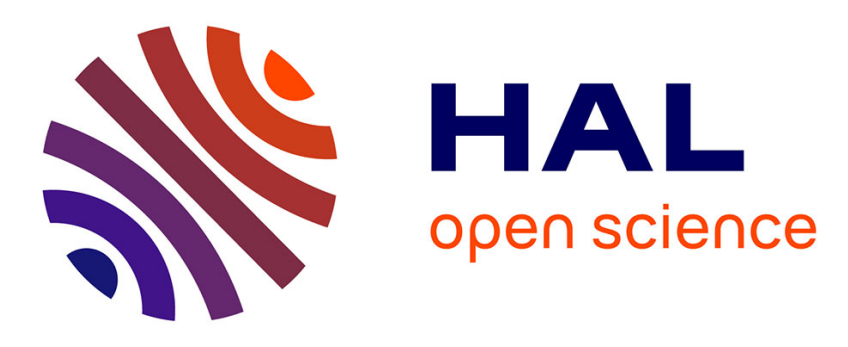

\title{
Estimating Relevance of Variables for Effective Recombination
}

Taishi Ito, Hernan Aguirre, Kiyoshi Tanaka, Arnaud Liefooghe, Bilel Derbel, Sébastien Verel

\section{- To cite this version:}

Taishi Ito, Hernan Aguirre, Kiyoshi Tanaka, Arnaud Liefooghe, Bilel Derbel, et al.. Estimating Relevance of Variables for Effective Recombination. EMO 2019 - International Conference on Evolutionary Multi-Criterion Optimization, Mar 2019, East Lansing, Michigan, United States. pp.411-423, 10.1007/978-3-030-12598-1_33. hal-02064547

\section{HAL Id: hal-02064547 https://hal.science/hal-02064547}

Submitted on 13 Sep 2021

HAL is a multi-disciplinary open access archive for the deposit and dissemination of scientific research documents, whether they are published or not. The documents may come from teaching and research institutions in France or abroad, or from public or private research centers.
L'archive ouverte pluridisciplinaire HAL, est destinée au dépôt et à la diffusion de documents scientifiques de niveau recherche, publiés ou non, émanant des établissements d'enseignement et de recherche français ou étrangers, des laboratoires publics ou privés. 


\title{
Estimating Relevance of Variables for Effective Recombination
}

\author{
Taishi Ito ${ }^{1,4}$, Hernán Aguirre ${ }^{1,4}$, Kiyoshi Tanaka ${ }^{1,4}$, \\ Arnaud Liefooghe ${ }^{2,4}$, Bilel Derbel ${ }^{2,4}$, and Sébastien Verel ${ }^{3,4}$ \\ 1 Shinshu University, Faculty of Engineering, Japan \\ \{17w2006d, ahernan, ktanaka\}@shinshu-u.ac.jp \\ 2 Université Lille 1, CRIStAL - Inria Lille-Nord Europe, France \\ \{arnaud.liefooghe, bilel.derbel\}@univ-lille.fr \\ 3 Université du Littoral Cote d'Opale, LISIC, France \\ verel@lisic.univ-littoral.fr \\ 4 International Associated Laboratory LIA-MODO
}

\begin{abstract}
Dominance, extensions of dominance, decomposition, and indicator functions are well-known approaches used to design MOEAs. Algorithms based on these approaches have mostly sought to enhance parent selection and survival selection. In addition, several variation operators have been developed for MOEAs. We focus on the classification and selection of variables to improve the effectiveness of solution search. In this work, we propose a method to classify variables that influence convergence and increase their recombination rate, aiming to improve convergence of the approximation found by the algorithm. We incorporate the proposed method into NSGA-II and study its effectiveness using three-objective DTLZ and WFG functions, including unimodal, multimodal, separable, non-separable, unbiased, and biased functions. We also test the effectiveness of the proposed method on a real-world biobjective problem. Simulation results verify that the proposed method can contribute to achieving faster and better convergence in several kinds of problems, including the real-world problem.
\end{abstract}

Keywords: Evolutionary multi-objective optimization - Variables classification · Variables selection · Recombination operators.

\section{Introduction}

Multi-objective evolutionary algorithms[4,2] (MOEAs) have been used to solve multi-objective optimization problems on all kinds of application domains. Due to their success, MOEAs are being applied to real-world problems of increased complexity. Scalability in decision and objective spaces, epistasis, effectiveness on problems with difficult topologies of the Pareto optimal set, and a limited budget of evaluations due to computationally expensive fitness functions are some of the challenges the new generation of MOEAs have to face.

The enhancement of MOEAs performance is an active research subject. Dominance, extensions of dominance, decomposition, and indicator functions are wellknown approaches used to design MOEAs[5], [10], [9], [11], [1]. Algorithms based 
on these approaches have mostly sought to enhance parent selection and survival selection. In addition, several variation operators have been developed and incorporated within MOEAs.

Evolutionary multi-objective algorithms commonly select individuals for variation based on their fitness. However, the operators of variation are commonly applied to variables randomly chosen. Typically, an operator rate per variable controls the expected number of variables that will be subject to variation, but the decision of what variables will be modified is left to chance. Modifying a variable of a solution in a multi-objective problem can have one of the following effects. The modification improves one or several objectives without worsening others. This would be the case if the solution subject to the modification is suboptimal, which is commonly observed in random initial populations and during early stages of the optimization. The modification improves one or more objectives but worsens others. This will typically be observed if the solution being modified is Pareto optimal or if it belongs to a local front. Multi-objective evolutionary algorithms aim to find an approximation of the Pareto optimal set, commonly with good qualities in terms of convergence and diversity in objective space. If the effects a variable has on convergence and diversity can be learned or estimated during the optimization[8], the effectiveness of the search could be enhanced by targeting particular variables for variation to find better approximations of the Pareto optimal set.

We focus on the classification and selection of variables for variation aiming to improve the ability of solution search. In this work, we propose a method to identify variables that influence convergence and increase their selection probabilities, so that recombination can select them more frequently to improve convergence of the approximation found by the algorithm. The proposed method selects randomly a solution from the instantaneous Pareto set and creates variations of it mutating one variable at the time. Variables are classified into influential and uninfluential based on whether there is a dominance relation or not between the original solution and the corresponding one-variable mutants. The method estimates that influential variables affect convergence of solutions in objective space and increase their recombination rate.

In this paper, we incorporate the proposed method into NSGA-II[5] and study its effectiveness using three-objective DTLZ[3] and WFG[6] functions, including unimodal, multimodal, separable, non-separable, unbiased, and biased functions. We also verify the effectiveness of the proposed method on a real-world biobjective problem [7]. Three ways to determine the trial values of variables to create the mutants are investigated. Simulation results verify that the proposed method can contribute to achieving faster and better convergence in several kinds of problems.

\section{Method}

In this section, we describe the proposed variable selection method, applied every generation after front sorting before truncation selection. The method first clas- 


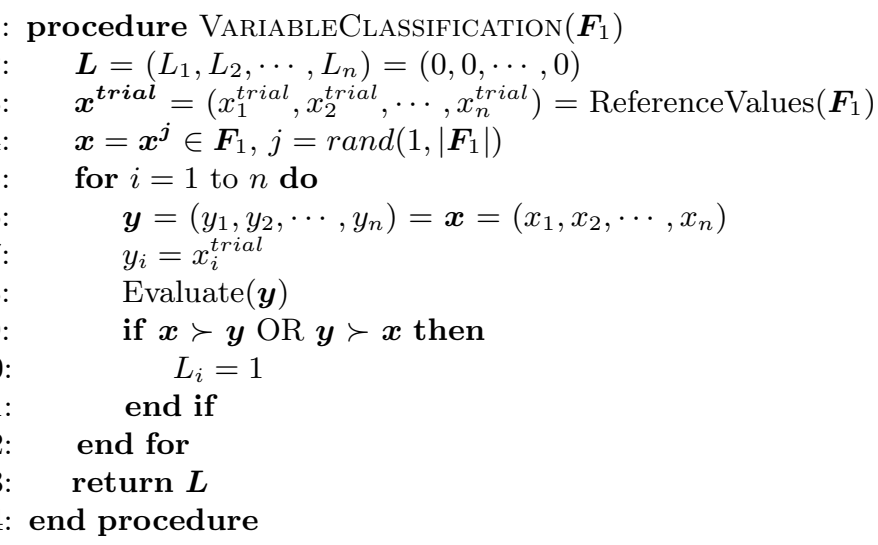

Fig. 1. Classification of variables

sifies variables that can influence convergence and then update their probabilities so that recombination can select them more frequently.

The procedure to classify variables is illustrated in the pseudocode of Figure 1. First, we assign a label 0 to each variable, $L_{i}=0, i=1, \cdots, n$. Next, we obtain trial values for each variable $\boldsymbol{x}^{\text {trial }}=\left(x_{1}^{\text {trial }}, \cdots, x_{n}^{\text {trial }}\right)$ from the non-dominated solution set $\boldsymbol{F}_{1}$ in the population $P_{t}$ at generation $t$. We randomly select one solution $\boldsymbol{x}=\left(x_{1}, \cdots, x_{n}\right)$ from $\boldsymbol{F}_{1}$. Then, for each variable $i$, we generate a solution $\boldsymbol{y}$ duplicate of $\boldsymbol{x}$ and modify the $i$-th variable with its corresponding trial value, $y_{i}=x_{i}^{\text {trial }}$. Evaluate $\boldsymbol{y}$ and calculate the dominance relation between solutions $\boldsymbol{y}$ and $\boldsymbol{x}$. If either $\boldsymbol{y}$ dominates $\boldsymbol{x}$ or $\boldsymbol{x}$ dominates $\boldsymbol{y}$, we update the corresponding label to $1, L_{i}=1$. This procedure returns the vector of labels $\boldsymbol{L}$ assigned to the variables, where $L_{i}=1$ if the change in the $i$-th variable induced a dominance relation $(\succ)$ between the randomly sampled solution and its one-variable mutant. $L_{i}=0$ otherwise.

We explore three procedures called random, far, and near to set trial values for variables.

random sets $x_{i}^{\text {trial }}$ to the value of the $i$-th variable of a solution randomly selected from $\boldsymbol{F}_{1}$. A different solution $j$ is randomly chosen for each variable $i$,

$$
x_{i}^{\text {trial }}=z_{i}^{j} \mid \boldsymbol{z}^{\boldsymbol{j}} \in \boldsymbol{F}_{1} \wedge j=\operatorname{rand}\left(1,\left|\boldsymbol{F}_{1}\right|\right) .
$$

far sets $x_{i}^{\text {trial }}$ to the farthest value of $x_{i}$ in $\boldsymbol{F}_{1}$. That is,

$$
\begin{aligned}
& k=\underset{j=1 \ldots\left|\boldsymbol{F}_{1}\right|}{\arg \max }\left(\left|x_{i}-z_{i}^{j}\right|\right), \quad \boldsymbol{z}^{j} \in \boldsymbol{F}_{1}, x_{i} \neq z_{i}^{j} \\
& x_{i}^{\text {trial }}=z_{i}^{k} .
\end{aligned}
$$

On the other hand, near sets $x_{i}^{\text {trial }}$ to the nearest value of $x_{i}$ in $\boldsymbol{F}_{1}$. That is,

$$
\begin{aligned}
& k=\underset{j=1 \ldots\left|\boldsymbol{F}_{1}\right|}{\arg \min }\left(\left|x_{i}-z_{i}^{j}\right|\right), \quad \boldsymbol{z}^{j} \in \boldsymbol{F}_{1}, x_{i} \neq z_{i}^{j} \\
& x_{i}^{\text {trial }}=z_{i}^{k} .
\end{aligned}
$$


In near and far, if all solutions in $\boldsymbol{F}_{1}$ have the same value in $x_{i}$, then $x_{i}^{\text {trial }}=$ $x_{i}$ (no change).

After variables have been classified, a recombination rate $p_{c v, i}^{\prime}$ for each variable $i$ is computed as follows

$$
\begin{aligned}
p_{c v, i}^{\prime} & =p_{c v}, \quad \text { if } \quad N_{L}=0 . \\
p_{c v, i}^{\prime} & =\left\{\begin{array}{c}
1, \quad \text { if } \quad L_{i}=1 \\
\frac{E-N_{L}}{n-N_{L}}, \text { otherwise, }
\end{array}, \quad \text { if } \quad 0<N_{L} \leq E .\right. \\
p_{c v, i}^{\prime} & =\left\{\begin{array}{c}
\frac{E}{N_{L}}, \text { if } \quad L_{i}=1 \\
0, \text { otherwise. }
\end{array}, \quad \text { if } \quad N_{L}>E .\right.
\end{aligned}
$$

where $L_{i} \in\{0,1\}$ is the label assigned to variables, $N_{L}=\sum_{i=1}^{n} L_{i}$ is the number of variables classified with label $L_{i}=1, E=n \times p_{c v}$ is the expected number of crossed variables using the default rate. If after exploring $n$ one-variable mutants no changes in dominance are observed, $N_{L}=0$, the recombination rate $p_{c v, i}^{\prime}$ is set to the default rate $p_{c v}$ for each variable. If there are changes in dominance and these are less than the expected number of crossed variables when the default crossover rate is used, $0<N_{L} \leq E$, then the recombination rate $p_{c v, i}^{\prime}$ is set to 1 for variables labeled $L_{i}=1$ and to $\frac{E-N_{L}}{n-N_{L}}<p_{c v}$ for variables labeled $L_{i}=0$. Otherwise, if $N_{L}>E, p_{c v, i}^{\prime}$ is set to $\frac{E}{N_{L}} \geq p_{c v}$ for variables labeled $L_{i}=1$ and to 0 for variables labeled $L_{i}=0$. Note that the expected number of recombined variables with $p_{c v}$ and $p_{c v}^{\prime}$ is the same.

In this work, we use the variable classification procedure to update recombination probabilities of variables. However, this method can be easily extended to influence probabilities for mutation or other variation operators.

\section{Test Problems}

DTLZ [3] and WFG [6] benchmark multi-objective optimization problems are used to evaluate the proposed method. These problems are scalable in the number of variables and the number of objective functions. From the DTLZ family of problems we use DTLZ2 and DTLZ3, whereas from the WFG family we use WFG1-WFG9. Some properties of these problems are summarized in Table 1. We include separability, modality and bias. Separable problems are marked with $\mathrm{S}$ and non-separable with NS. Unimodal problems are marked U and multimodal problems with $\mathrm{M}$.

We also test the effectiveness of the proposed method on a bi-objective realworld problem [7]. The problem is to design a platform with a motor mounted on it. The machine setup is simplified as a pin-pin supported beam carrying a weight (motor). A vibratory disturbance is imparted from the motor onto the beam, which is of length $L$, width $b$, and symmetrical about its mid-plane. The beam is made of three layers of material. Variables $d_{1}$ and $d_{2}$, respectively, locate the contact of materials 1 and 2 , and 2 and 3 . Variable $d_{3}$ locates the top of the beam. The values of $d_{1}, d_{2}$, and $d_{3}$ are measured from the mid-plane 
Table 1. Features of Test Problems. Separability: separable S, non-separable NS. Modality: unimodal U, multimodal M.

\begin{tabular}{lcccc}
\hline Problem & Sep. Modality Bias & Other Features \\
\hline DTLZ2 & $\mathrm{S}$ & $\mathrm{U}$ & - & \\
DTLZ3 & $\mathrm{S}$ & $\mathrm{M}$ & - & \\
WFG1 & $\mathrm{S}$ & $\mathrm{U}$ & Yes Polynomial bias $\alpha=0.02$. Bias variables towards 1 \\
WFG2 & $\mathrm{NS}$ & $\mathrm{U}, \mathrm{M}$ & - & $\mathrm{U}: f_{1}, \cdots, f_{M-1}, \mathrm{M}: f_{M}, f_{M}$. Discontinuous front \\
WFG3 & $\mathrm{NS}$ & $\mathrm{U}$ & - & Degenerancy constants $A_{1}=1, A_{2: M-1}=0$, \\
& - & & & front reduces to two dimensions \\
WFG4 & $\mathrm{S}$ & $\mathrm{M}$ & - & \\
WFG5 & $\mathrm{S}$ & $\mathrm{M}$ & - & \\
WFG6 & $\mathrm{NS}$ & $\mathrm{U}$ & - & \\
WFG7 & $\mathrm{S}$ & $\mathrm{U}$ & Yes Parameter dependent bias: $z_{i=1: k} \leftarrow z_{i+1}, \ldots, z_{n}$ \\
WFG8 & $\mathrm{NS}$ & $\mathrm{U}$ & Yes Parameter dependent bias: $z_{i=k+1: n} \leftarrow z_{1}, \ldots, z_{i-1}$ \\
WFG9 & $\mathrm{NS}$ & $\mathrm{M}$ & Yes Parameter dependent bias: $z_{i=1: n-1} \leftarrow z_{i+1}, \ldots, z_{n}$ \\
\hline
\end{tabular}

of the beam. $M_{i}$ refers to the material type for layer $i(i=1,2,3)$. The mass density $(\rho)$, Young's modulus of elasticity $(E)$, and cost per unit volume $(c)$ for each material type is given [7]. The objective functions are the fundamental frequency, $f_{1}$, to be maximized and the cost of the set up, $f_{2}$, to be minimized. The complete formulation is as follows,

$$
\begin{aligned}
f_{1}\left(d_{1}, d_{2}, d_{3}, b, L\right) & =\left(\pi / 2 L^{2}\right)(E I / \mu)^{1 / 2}, \\
E I & =(2 b / 3)\left[E_{M_{1}} d_{1}^{3}+E_{M_{2}}\left(d_{2}^{3}-d_{1}^{3}\right)+E_{M_{3}}\left(d_{3}^{3}-d_{2}^{3}\right)\right], \\
\mu & =2 b\left[\rho_{M_{1}} d_{1}+\rho_{M_{2}}\left(d_{2}-d_{1}\right)+\rho_{M_{3}}\left(d_{3}-d_{2}\right)\right], \\
f_{2}\left(d_{1}, d_{2}, d_{3}, b\right) & =2 b\left[c_{M_{1}} d_{1}+c_{M_{2}}\left(d_{2}-d_{1}\right)+c_{M_{3}}\left(d_{3}-d_{2}\right)\right],
\end{aligned}
$$

subject to $\mu L-2800<0, d_{2}-d_{1} \leq 0.01, d_{3}-d_{2} \leq 0.01,0.35 \leq b \leq 0.5$, $3 \leq L \leq 6$, and $d_{1}, d_{2}, d_{3} \in[0.01,0.6]$.

\section{Experimental Setup and Performance Measures}

In this paper, we use DTLZ2, DTLZ3, WFG1-WFG9 as benchmark problems, setting the number of objectives $M=3$ and the number of variables $n=12$ for all problems. The number of position variables is $M-1$ in DTLZ. Similarly, we set the number of position variables to $k=M-1$ in WFG. Thus, the number of position variables is 2 and the number of distance variables is 10 in all benchmark problems dealt with in this paper.

We use five algorithms to study and verify the performance of the variable selection method proposed in this work. The base algorithm is conventional NSGA-II [5]. NSGA-II randomly selects variables for recombination with the same probability $p_{c v}$ per variable. In the following, NSGA-II is named org. We also use three variations of NSGA-II using the proposed variable selection 
method with one of the procedures to set trial variables, as explained in section 2. These variations are named random, far, and near. The fifth algorithm, named ideal, knows in advance the correct classification of distance and position variables to compute the recombination rate per variable. Thus, org provides a baseline for performance comparison, whereas ideal provides the performance reference of an algorithm with a perfect classification of variables.

The algorithms are run for 2000 generations setting the number of individuals to 100 , the recombination rate per individual to 1.0 , and the mutation rate to $1 / n$. The recombination rate per variable is set to $p_{c v}=0.5$ in org and to $\boldsymbol{p}_{\boldsymbol{c} v}^{\prime}$ in random, far, near, and ideal, computed with Eq. (4-6) as explained in section 2. The number of runs is 30 in all experiments.

To verify improvements on convergence we use generational distance GD in the case of DTLZ problems and the value of variable $x_{M}$ in WFG problems. GD is computed analitycally. The smaller the value of GD, the better the convergence of the set of obtained nondominated solutions. In WFG distance variables are finally aggregated into variable $x_{M} \in[0.0,1.0]$. The smaller the value of $x_{M}$ is, the closer to the true optimum the solution is. When $x_{M}=0$, the obtained solution is the true optimal solution. We also use the hypervolume metric to evaluate performance in the real world problem.

\section{Simulation Results and Discussion}

\subsection{Three-objective Benchmark Problems}

First, we apply each algorithm to DTLZ2 and DTLZ3 functions. Figure 2 (a) shows the transition of the average GD value in 30 runs over the number of fitness evaluations for DTLZ2. Similarly, Figure 2 (b) shows results for DTLZ3. In this experiment, population size is set to 100 and the number of generations to 2,000 . Therefore, the total number of evaluations in org and ideal is 200,000 per run. In the case of random, far, and near the algorithms evaluate an additional trial solution per variable. Since the number of variables is 12 , random, far, and near evaluate 112 solutions in each generation and 224,000 evaluations per run.

Comparing GD by the algorithms in Figure 2 (a) and (b), it can be seen that random, far, and near approach ideal and obtain significantly smaller GD than org in both separable problems, the unimodal DTLZ2 and in the multimodal DTLZ3. In DTLZ2, no significant difference is observed among random, far, and near. In the multimodal DTLZ3, during the latest stages of the search, far seems to perform better than random and near, in that order. Note that there is one order of magnitude difference between far and org in DTLZ2 and two orders of magnitude difference in DTLZ3.

Next, we apply each algorithm to WFG1-WFG9 problems. Figure 3 shows the transition of the median value of the distance variable $x_{M}$ in the set of nondominated solutions over the number of evaluations. Comparing the algorithms, it can be seen that random, far, and near approach ideal and achieve smaller 


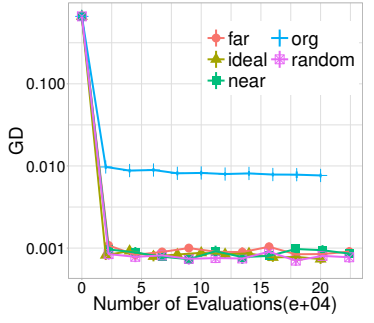

(a) DTLZ2

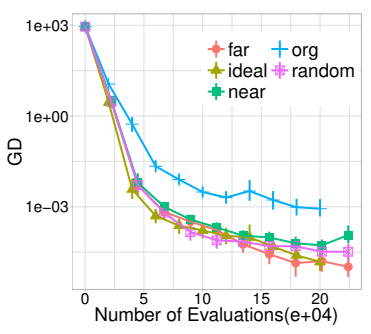

(b) DTLZ3

Fig. 2. Transition of the median value of GD over the number of fitness evaluations in DTLZ2 and DTLZ3 problems

$\mathrm{m}$

$x_{M}$ values than org in WFG1, WFG2, WFG4, WFG5, WFG6, and WFG8, improving significantly convergence. In WFG3, WFG7, and WFG9 $x_{M}$ value is similar for all algorithms.

Table 2. Rate of absolute classification of all variables.

\begin{tabular}{l|ccccccccccc}
\hline & DTLZ2 & DTLZ3 & WFG1 & WFG2 & WFG3 & WFG4 & WFG5 & WFG6 & WFG7 & WFG8 & WFG9 \\
\hline far & 99.6 & 76.6 & 38.0 & 99.9 & 34.6 & 99.7 & 99.9 & 100 & 4.06 & 98.1 & 0.805 \\
\hline near & 71.6 & 43.1 & 14.9 & 92.3 & 34.6 & 98.3 & 69.5 & 99.2 & 6.41 & 91.3 & 0.485 \\
\hline random & 86.9 & 53.6 & 13.3 & 86.7 & 30.8 & 89.6 & 83.5 & 89.6 & 2.78 & 86.7 & 0.240 \\
\hline
\end{tabular}

Table 2 shows the average rate of absolute classification of variables. A classification is counted as absolute when all position and distance variables are correctly classified. From Table 2 , it can be seen that the rates of absolute classification are high in problems DTLZ2, WFG2, WFG4-6, and WFG8, where convergence improves in the algorithms with the proposed method as shown in Figure 2 and Figure 3. Absolute classification rate is low in problems WFG3, WFG7, and WFG9, were convergence did not improve. Note that absolute classification is low in DTLZ3 and WFG1, although the proposed method significantly improves convergence.

To analyze with more detail the classification of variables and its impact on performance, Figure 4 shows the classification rate per variables over the generations for some problems. For each variable, we compute the percentage it was assigned label 1 in every 100 generations.

In all DTLZ and WFG problems, the classification rates of position variables $x_{1}, x_{2}$ and $z_{1}, z_{2}$ are 0 in nearly all generations. In WFG8, the classification rate of distance variables $x_{3}, \ldots, x_{12}$ and $z_{3}, \ldots, z_{12}$ are 1 in almost all generations. A similar situation occurs in problems DTLZ2, WFG2, and WFG4-6 (not shown here). As shown in Figure $\mathbf{2}$ and Figure 3, the convergence performance im- 


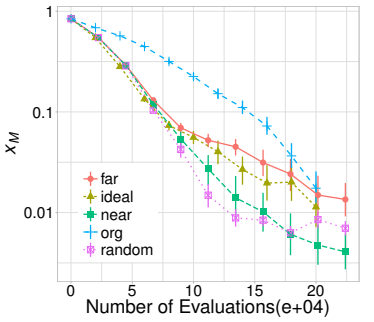

(a) WFG1

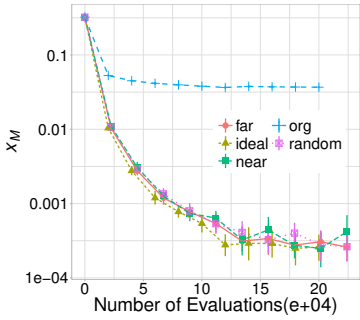

(d) WFG4

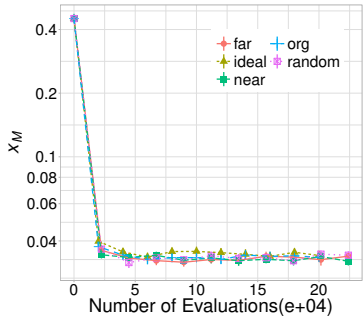

(g) WFG7

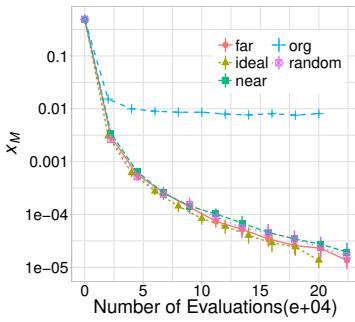

(b) WFG2

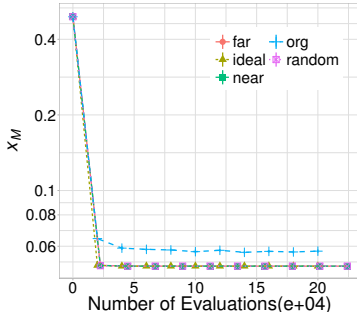

(e) WFG5

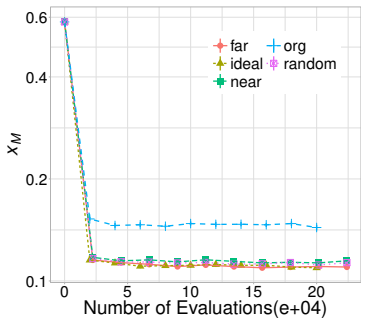

(h) WFG8

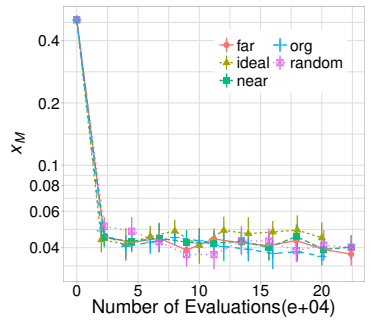

(c) WFG3

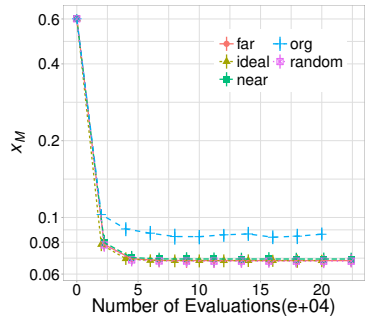

(f) WFG6

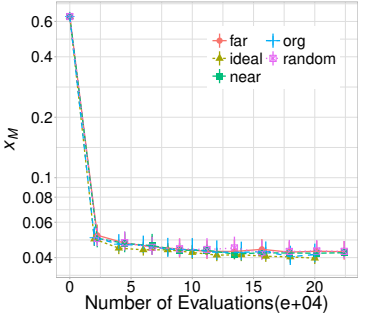

(i) WFG9

Fig. 3. Transition of the median value of the distance variable $x_{M}$ over the number of fitness evaluations in WFG problems

proved in the proposed methods compared with the conventional NSGA-II in problems where classification rates of the distance variable are high. In these problems, it is considered that the convergence performance has improved since the distance variables are estimated correctly and they are searched intensively.

Looking at DTLZ3 and WFG1 in Figure 4, distance variables were correctly classified in the early generations, but their classification rate decreases in later generations. This is because, in these problems, the value of distance variable tends to converge to the same value, i.e. solutions are trapped in local optima. When this happens, the trial value of the variable in the mutant is the same as the value of the variable in the original solution and therefore a dominance relation does to occur between them. From Figure 2 and Figure 3, DTLZ3 


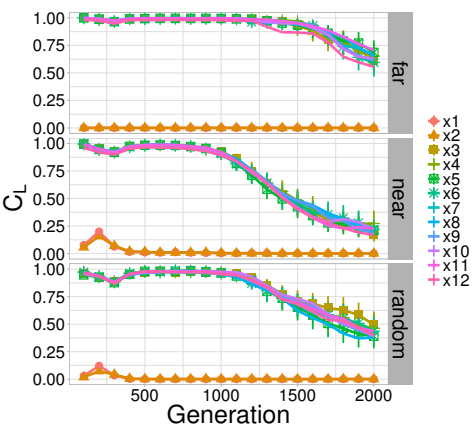

(a) DTLZ3

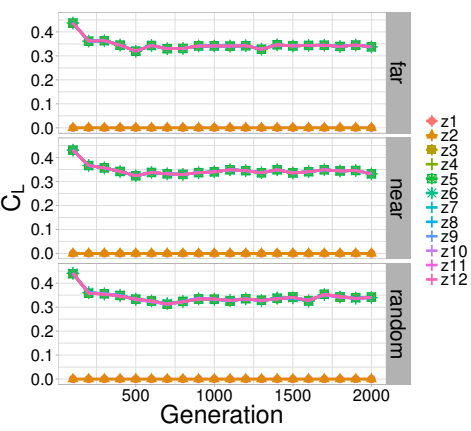

(c) WFG3

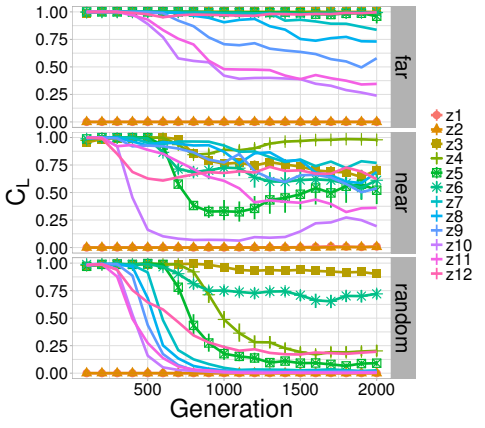

(b) WFG1

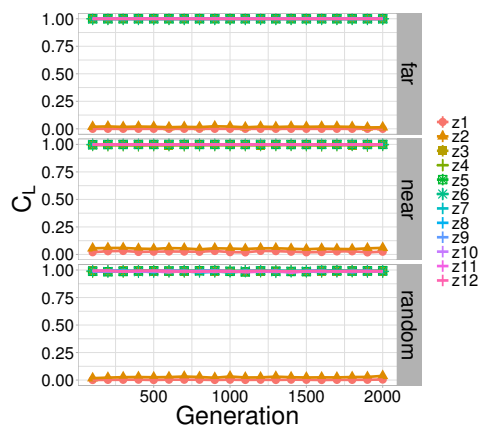

(d) WFG8

Fig. 4. Classification rate per variables over the generations

and WFG1, in which distance variables were estimated correctly in the early generations, distance variable converged to values close to optimal in all three proposed methods, so the convergence performance of the solution improved compared with the conventional method. Note that in WFG1, with the passing of generations, the rate of correct classification of distance variables is gradually higher from $z_{3}$ to $z_{12}$. This is because the influence of the distance variables of WFG1 on the convergence of the solution increases from $z_{3}$ to $z_{12}$.

Note from Figure 4 that the classification rate of the distance variables in WFG3 was low throughout the generations. Something similar happens in problems WFG7 and WFG9. In these problems, distance variables affect position variables as indicated in the Other Features column of Table 1. In WFG3, when the value of a distance variable changes, the value of the position variable changes as well due to degeneracy after the transition. In the case of WFG7 $\left(z_{i=1: k} \leftarrow z_{i+1}, \ldots, z_{n}\right)$ and WFG9 $\left(z_{i=1: n-1} \leftarrow z_{i+1}, \ldots, z_{n}\right)$, a transformation is applied whereby the value of the distance variable is used to bias the value of position variables. As shown in Figure 3, the convergence performance of the proposed method on these problems did not differ from the conventional method 


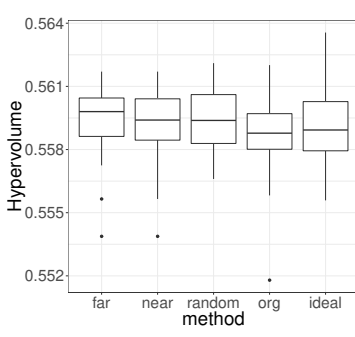

(a) WFG3

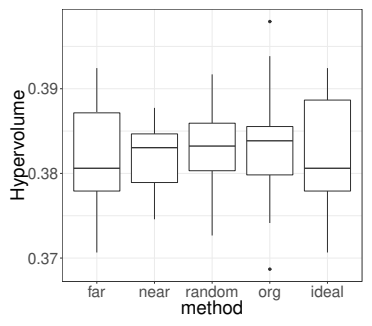

(b) WFG5

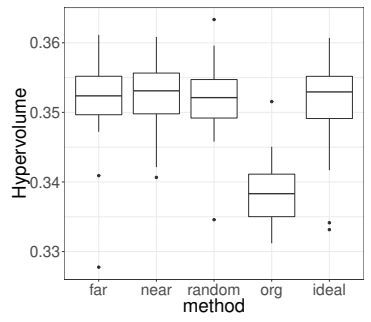

(c) WFG8

Fig. 5. Hypervolume

and ideal. In these problems, finally note that the classification rate of distance variable was higher in near than random and far. This is probably because the change of the value of the distance variable was small and the values of position variables did not change greatly.

When distance variables also affect position variables, a dominance relation between a solution and its one-variable mutant is less likely to occur and therefore becomes more difficult to classify distance variables in these problems. Note that linkage can be very large in these problems, where some variables are affected by almost all other variables, or some variables affect most other variables. It is worth studying with more detail the effects of linkage on variables identification.

On the other hand, note that variables are correctly classified when position variables affect distance variables $\left(z_{i=k+1: n} \leftarrow z_{1}, \ldots, z_{i-1}\right)$, and convergence can be improved as shown by the results on WFG8.

Modality and non-separability of a function seem not to affect the correct classification of variables. Note that there is a high classification rate for unimodal and multi-modal problems, separable and non-separable, when distance variables do not affect the position of solutions. Also, linkage between distance variables and linkage from position to distance variables are not an issue for correct classification.

In this work, the proposed method favors recombination of variables that can improve convergence. However, in addition to convergence, the aim of a multi-objective optimizer is to also achieve a set of well-distributed solutions. To verify whether the proposed method has a negative impact on diversity we also compute the hypervolume of the solutions found by the algorithms, which measures both convergence and diversity. Figure $\mathbf{5}$ shows the hypervolume for WFG3, WFG5, and WFG8. In general, where there is a clear improvement in convergence we also observe an improvement in hypervolume, as illustrated in Figure 3 (h) and Figure 5 (c) for WFG8. In cases where there is no difference in convergence or is very small, hypervolume is also similar, as shown in Figure 3 (c) and Figure 5 (a) for WFG3 and Figure $\mathbf{3}$ (e) and Figure 5 (b) for WFG5. These results suggest that there is not a serious detriment to diversity of solutions. However, emphasizing variation of variables that improve diversity 


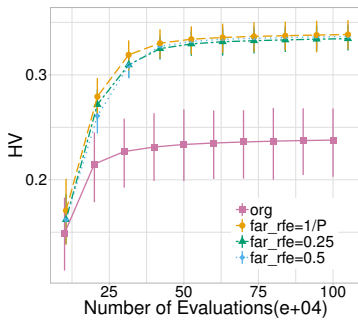

(a) Hypervolume

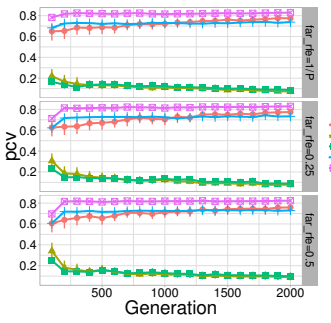

(b) Pcv

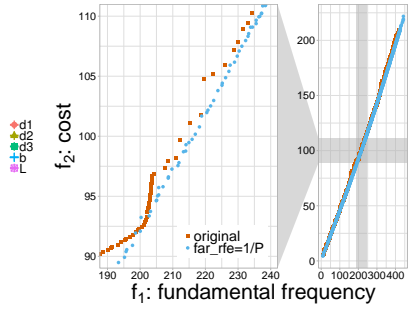

(c) Pareto front

Fig. 6. Results on the vibrating beam problem

would enhance further the performance of the multi-objective algorithm. In the future, we would like to extend the method to focus on diversity as well.

Summarizing, the convergence performance of the proposed method on benchmark problems with random, far, or near procedures to set the trial variables improves compared to org, because distance variables are correctly estimated and the frequency of recombining them is increased.

\subsection{Vibrating Beam Problem}

This bi-objective problem consists of 5 variables and 3 constraints. A random initialization of the population in this problem leads to all solutions being unfeasible. Approximately, 3 in every 10.000 randomly created solutions is feasible. However, a simple constraint handling method allows the algorithm fo find feasible solutions after 5 or 6 generations (500-600 function evaluations) and accumulate them thereafter. Around generation 20 all solutions in the population are feasible. Figure 6 shows results by NSGA-II with the constraint handling technique, denoted org, and by the proposed method far applied after 1 solution is feasible, $25 \%$ and $50 \%$ of the population is feasible. Note from Figure 6 (a) that the hypervolume by the proposed method is significantly higher. Also, note from Figure 6 (b) that variables $d_{1}, b$ and $L$ are consistently classified as influential for convergence and their probability $p_{c v}$ for recombination is higher. From Figure 6 (c) note that the proposed method improves convergence towards the Pareto front.

\section{Conclusions}

In this work we have proposed a method to classify variables that influence convergence and increased their selection probabilities to recombine them more often. The classification procedure is based on whether there is a Pareto dominance relation between one-variable mutants. The proposed method was tested 
on DTLZ and WFG functions, including unimodal, multimodal, separable, nonseparable, unbiased and biased functions. Our experimental results show that the proposed method can improve significantly the performance of the well known NSGA-II algorithm in most instances for 3 objective functions. Modality and non-separability of a function seem not to affect the correct classification of variables. Also, linkage between distance variables and linkage from position to distance variables are not an issue for correct classification. However, performance could not be improved in problems where linkage from distance to position variables is very large. We also verified that the proposed method can improve convergence without affecting diversity on a real world bi-objective problem. In the future we would like to study with more detail the effect of linkage. Also, we would like to study the scalability in objective space, particularly since we use a dominance relation to classify variables. In addition, we would like to study ways to extend the proposed method for large scale problems.

\section{References}

1. Aguirre, H., Oyama, A., Tanaka, K.: Adaptive $\varepsilon$-sampling and $\varepsilon$-hood for evolutionary many-objective optimization. In: Evolutionary Multi-Criterion Optimization. Lecture Notes in Computer Science, vol. 7811, pp. 322-336 (2013)

2. Coello, C.C., Lamont, G., van Veldhuizen, D.: Evolutionary Algorithms for Solving Multi-Objective Problems. Genetic and Evolutionary Computation, Springer, Berlin, Heidelberg, 2nd edn. (2002)

3. Deb, K., Thiele, L., Laumanns, M., Zitzler, E.: Scalable multi-objective optimization test problems. In: Congress on Evolutionary Computation. pp. 825-830. IEEE Service Center (2002)

4. Deb, K.: Multi-Objective Optimization using Evolutionary Algorithms. John Wiley \& Sons (2001)

5. Deb, K., Agrawal, S., Pratap, A., Meyarivan, T.: A fast elitist non-dominated sorting genetic algorithm for multi-objective optimisation: NSGA-II. In: Proceedings Parallel Problem Solving from Nature - PPSN VI. vol. 1917, pp. 849-858 (2000)

6. Huband, S., Hingston, P., Barone, L., While, R.: A review of multi-objective test problems and a scalable test problem toolkit. IEEE Transactions on Evolutionary Computation 10(5), 477-506 (2007)

7. Narayanan, S., Azarm, S.: On improving multiobjective genetic algorithms for design optimization. Structural optimization 18(2), 146-155 (Oct 1999)

8. Sagawa, M., Aguirre, H., Daolio, F., Liefooghe, A., Derbel, B., Verel, S., Tanaka, K.: Learning variable importance to guide recombination. In: IEEE SSCI. pp. 1-7 (2016)

9. Zhang, Q., Li, H.: MOEA/D: A multiobjective evolutionary algorithm based on decomposition. IEEE Trans. Evolutionary Computation 11(6), 712-731 (2007)

10. Zitzler, E., Laumanns, M., Thiele, L.: SPEA2: Improving the strength pareto evolutionary algorithm for multiobjective optimization. In: Evolutionary Methods for Design Optimization and Control with Applications to Industrial Problems. pp. 95-100. International Center for Numerical Methods in Engineering (2001)

11. Zitzler, E., Künzli, S.: Indicator-based selection in multiobjective search. In: Parallel Problem Solving from Nature - PPSN VIII. pp. 832-842. Springer Berlin Heidelberg, Berlin, Heidelberg (2004) 